\title{
Shear Modulus Effect on Acoustically Transparent Materials
}

\author{
PIETER S. DUBBELDAY AND KURT M. RITTENMYER
}

\begin{abstract}
Materials have been developed that match density $\rho$ and dilatational sound speed $c$ to the values of water as closely as possible while maintaining sufficient rigidity to serve for structural purposes. Matching of density and speed results in transparency for fluids only; the shear modulus in a solid admits the presence of a shear wave which causes deviation from ideal $\rho c$ behavior. The effect of a finite shear modulus on the reflection of plane waves by an infinite plane is analyzed. The shear modulus of the material was measured following a method developed by R. L. Adkins in 1966. Examples are given of the reflection coefficient as a function of incidence angle for values of $\rho$ and $c$ close to those of the medium, and various ratios of plate thickness to dilatational wavelength. The condition of total reflection is shown to be related to the occurrence of thickness-shear resonances. Insertion-loss measurements on a structurally rigid $\rho c$ panel are compared with results of the analysis.
\end{abstract}

\section{INTRODUCTION}

$\mathbf{P}$ UBLICATIONS [1], [2] have appeared in recent years that report on the development of an acoustically transparent material based on a fluoroepoxy filled with microballoons. Such a material can be designed to have a density $\rho_{s}$ and dilatational sound speed $c_{d}$ that closely match those of the acoustic medium, usually seawater, but with sufficient rigidity to serve as a material for structural members or backing plates of acoustical systems. The matching of density and dilatational sound speed leads to perfect acoustic transparency for reflection of sound at the interface $\mathrm{e}^{\mathrm{f}}$ fluids only. A solid has a finite shear modulus; and for other than perpendicular incidence, a shear wave will be present in addition to the dilatational wave. This shear wave causes a deviation from ideal transparency.

In this study (expanding on earlier work [3]) an analysis is given of the influence of the shear modulus on the reflection and transmission of a plane sound wave by an infinite plate with strictly or approximately matching density and sound speed. First the analysis is presented of reflection and transmission coefficients for an infinite plate, and examples are given for ideal and close-to-ideal $\rho c$ materials. Then the condition of total reflection is derived and its connection with thickness-shear resonances established. A comparison is made of insertion-loss measurements on a structurally rigid $\rho c$ panel with results of the analysis.

\section{ANALysis}

An early analysis of the reflection and transmission of a plane sound wave by an infinite plate was given by Reissner

Manuscript received June 15, 1986; revised November 25, 1986. This work was supported by the Office of Naval Research.

The authors are with the Underwater Sound Reference Detachment, Naval Research Laboratory, Orlando, FL 32856-8337.

IEEE Log Number 8714627.
[4], using exact elasticity theory. A more recent account may be found in the monograph by Brekhovskikh [5], who also discusses the thin-plate approximation to this problem. A similar analysis is presented in [6], closely following the approach and notation of Viktorov [7]. The emphasis here is on the application to $\rho c$ materials. The relevant definitions of [6] are repeated below.

A fluid with density $\rho_{0}$ is present at both sides of the plate (thickness $h=2 d$ ), Fig. 1. The incident wave arrives with angle $\theta$ to the normal, wave speed $c_{0}$, and wavenumber $k_{0}$. The transmitted wave forms an angle $\theta$ with the normal. The propagation of waves in the solid, excited by the incoming wave, is treated by exact elasticity theory. The straight-crested wave in the solid propagating in the $x$ direction with wavenumber $k$ is a combination of a dilatational wave, represented by a scalar potential $\phi$ with amplitudes $A_{s}$ and $B_{a}$ for the symmetric and antisymmetric contributions, respectively, and a shear wave represented by the only nonzero component of a vector potential $\psi$ with amplitudes $D_{s}$ and $C_{a}$. Other field variables are the pressure amplitudes of the incoming wave $P_{i}$, of the transmitted wave $P_{t}$, and of the reflected wave $P_{r}$. At the boundaries of the plate with the two fluids, one imposes conditions of continuous normal stress and velocity and zero tangential stress. Dependence of the field variables on time and $x$ direction parallel to the plate is expressed through the factor exp $i(\omega t-k x)$, which is subsequently suppressed.

The wavenumber $k$ of straight-crested waves in the plate is related to the wavenumber $k_{0}$ in the medium by the coincidence relation $k=k_{0} \sin \theta$. New pressure amplitudes are introduced by $P_{0}=P_{i} \exp \left(i k_{0} d \cos \theta\right), P=P_{r} \exp \left(-i k_{0} d\right.$ $\cos \theta$ ), and $P^{\prime}=P_{l} \exp \left(-i k_{0} d \cos \theta\right)$. Further, $\left(q^{\prime}\right)^{2}=k_{d}^{2}$ - $k^{2}$, and $\left(s^{\prime}\right)^{2}=k_{s}^{2}-k^{2}$, where $k_{d}$ and $k_{s}$ are the wavenumbers of the dilatational and shear waves in the solid; the corresponding wave speeds are $c_{d}$ and $c_{s}$.

Imposing the boundary conditions mentioned above leads to six linear equations (given in [6]) for the seven amplitudes $A_{s}$, $B_{a}, C_{a}, D_{s}, P_{0}, P$, and $P^{\prime}$. Repeated application of Cramer's rule enables one to express six of these amplitudes in terms of one selected one, say $P_{0}$. The ratios of the amplitudes $P$ and $P^{\prime}$ to $P_{0}$ are directly related to the reflection and transmission coefficients $R$ and $T$ :

$$
\begin{aligned}
& R=\frac{P_{r}}{P_{i}}=\frac{P}{P_{0}} \exp \left(2 i k_{0} d \cos \theta\right) \\
& T=\frac{P_{t}}{P_{i}}=\frac{P^{\prime}}{P_{0}} \exp \left(2 i k_{0} d \cos \theta\right)
\end{aligned}
$$




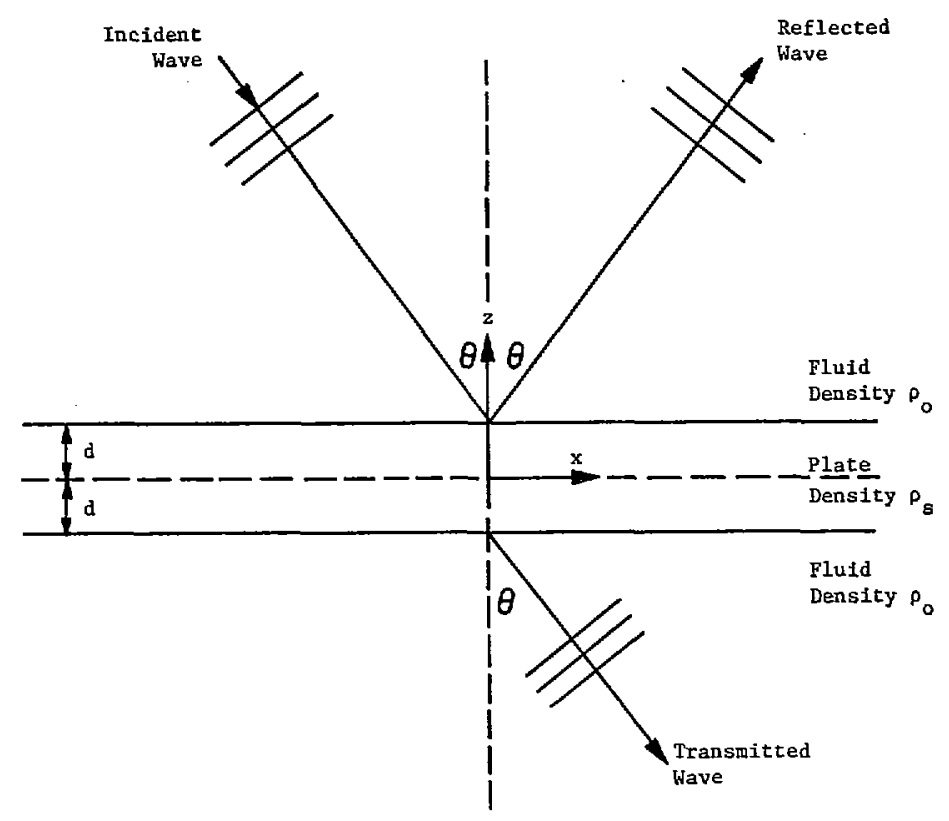

Fig. 1. Geometry of reflection and transmission of plane waves by infinite plate.

where

$$
\begin{aligned}
& \frac{P}{P_{0}}=\frac{-A+D}{A+B-C+D} \\
& \frac{P^{\prime}}{P_{0}}=\frac{B+C}{A+B-C+D}
\end{aligned}
$$

$A=\frac{1}{2}\left(\frac{\rho_{0}}{\rho_{s}}\right)^{2}\left(q^{\prime} d\right)^{2}\left(k_{s} d\right)^{4} \cos q^{\prime} d$

- $\sin q^{\prime} d \cos s^{\prime} d \sin s^{\prime} d$

$B=\frac{1}{2} i\left(\frac{\rho_{0}}{\rho_{s}}\right) k_{0} d q^{\prime} d \Delta_{s} \cos q^{\prime} d \cos s^{\prime} d \cos \theta$

$$
C=\frac{1}{2} i\left(\frac{\rho_{0}}{\rho_{s}}\right) k_{0} d q^{\prime} d \Delta_{a} \sin q^{\prime} d \sin s^{\prime} d \cos \theta
$$

$$
D=\frac{1}{2}\left(k_{0} d\right)^{2} \Delta_{a} \Delta_{s} \cos ^{2} \theta /\left(k_{s} d\right)^{4}
$$

and

$$
\begin{gathered}
\Delta_{a}=-\left(k^{2}-s^{\prime 2}\right)^{2} d^{4} \sin q^{\prime} d \cos s^{\prime} d \\
+4(k d)^{2} q^{\prime} d s^{\prime} d \cos q^{\prime} d \sin s^{\prime} d \\
\begin{aligned}
\Delta_{s}=-\left(k^{2}-s^{\prime 2}\right)^{2} d^{4} \cos q^{\prime} d \sin s^{\prime} d \\
+4(k d)^{2} q^{\prime} d s^{\prime} d \sin q^{\prime} d \cos s^{\prime} d .
\end{aligned}
\end{gathered}
$$

It may be noted that $\Delta_{a}=0$ and $\Delta_{s}=0$ are the dispersion relations for Lamb waves in an unloaded plate, antisymmetric and symmetric, respectively, [7].

\section{Thin-Plate Results}

Fig. 2 shows the reflection coefficient as a function of incidence angle $\theta$ for various values of relative density and sound speed. The three figures arranged along a column have equal values of $\rho_{0} / \rho_{s}$, namely, $0.995,1.000$, and 1.005 . The three figures arranged in a row have equal values of $c_{0} / c_{d}$ : $0.995,1.000$, and 1.005 . The plate is thin in terms of the dilatational wavelength $\lambda_{d}$. The three curves $1,2,3$ correspond to $h / \lambda_{d}=0.08,0.04,0.02$, respectively. Preliminary measurements of the shear modulus $G$ and dilatational wave speed $c_{d}$ implied $G / K=0.13$ ( $K$ is the bulk modulus), and this value was used in Figs. 2-5. The loss tangent $\eta$, defined as the ratio of the imaginary to the real part of $G$ is assumed zero in Figs. 2-4. The bulk modulus is assumed lossless. The value of Poisson's ratio $\nu$ corresponding to $G / K=0.13$ is found to be 0.438 .

One observes that for intermediate angles (between about $30^{\circ}$ and $60^{\circ}$ ) the appearance of all the curves is very similar, but with an increase in the maximum near $60^{\circ}$ with increasing $c_{0} / c_{d}$, and a bit less so with increasing $\rho_{0} / \rho_{s}$. For $\theta$ between $0^{\circ}$ and $30^{\circ}$ there is corresponding behavior for curves in figures lying on lines parallel to the main diagonal of the mosaic (Fig. 2). On the main diagonal (Fig. 2(c), (e), (g)), the curves go through the origin; above the diagonal (Fig. 2(a), (b), (d)), the curves have a zero reflection while below the diagonal (Fig. $2(\mathrm{f}),(\mathrm{h}),(\mathrm{i}))$, there is no zero reflection near $0^{\circ}$. Obviously the value of $\rho c$ is closely constant on lines parallel to the diagonal, so this behavior reflects the fact that the reflection near $\theta=0$ is governed by the product $\rho c$. Between $60^{\circ}$ and $90^{\circ}$ the figures with the same value of $\rho_{0} / \rho_{s}$ are similar; the value of $c_{0} / c_{d}$ governs the appearance of zero reflection: $c_{0} / c_{d}$ $<1$ zero reflection near $\theta=90^{\circ}, c_{0} / c_{d}=1$ zero reflection for $\theta=90^{\circ}, c_{0} / c_{d}>1$ no zero reflection near $\theta=90^{\circ}$.

\section{Condition for Total Reflection}

In Fig. 2 the plate thickness was considered relative to the dilatational wavelength since this wavelength follows as a direct consequence of the concern of matching $c_{0}$ and $c_{d}$ in rigid $\rho c$ materials. Further analysis shows that the shear wavelength $\lambda_{s}$ determines the behavior of thick plates rather than $\lambda_{d}$. 


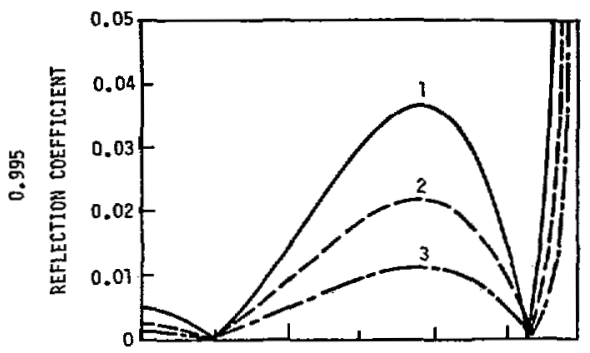

(a)

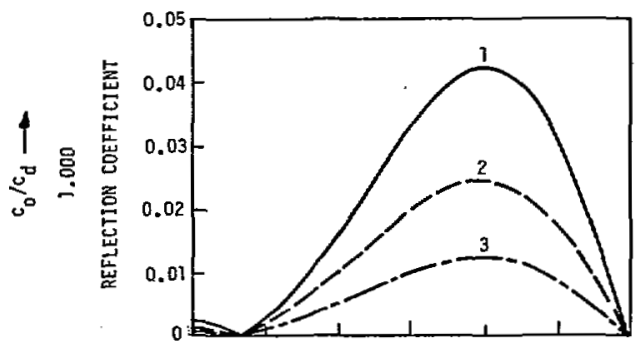

(d)

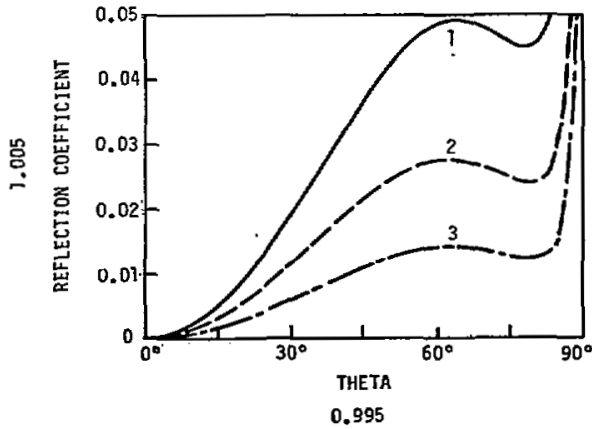

(g)

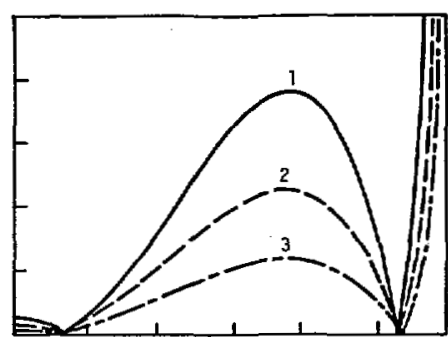

(b)

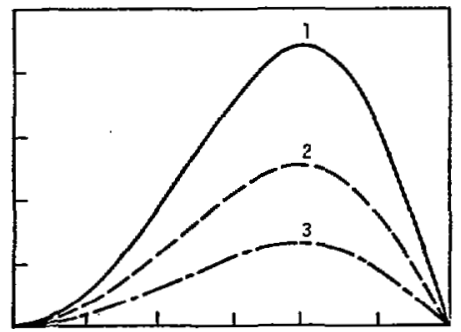

(e)

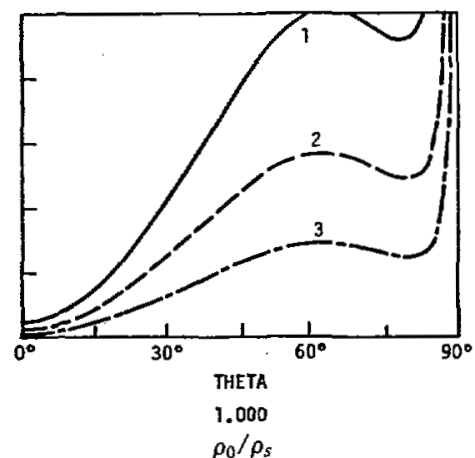

(h)

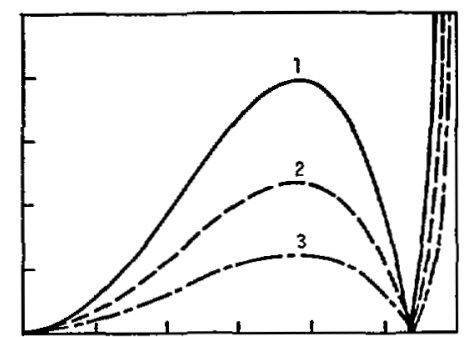

(c)

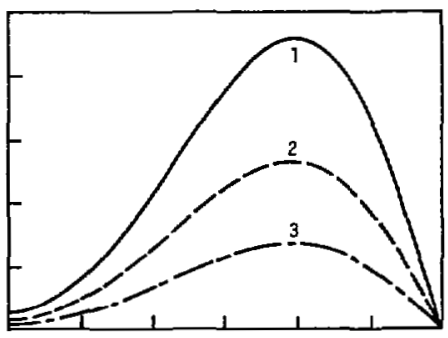

(f)

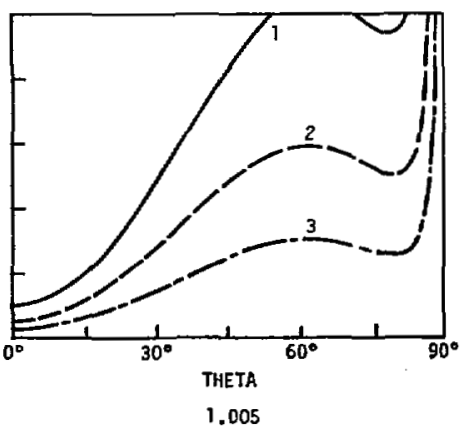

(i)

Fig. 2. (a)-(i) Reflection coefficient as a function of incidence angle for ideal and close-to-ideal rigid $\rho c$ materials. $G / K=0.13$, $\eta=0, \nu=0.438 .1-h / \lambda_{d}=0.08 ; 2-h / \lambda_{d}=0.04 ; 3-h / \lambda_{d}=0.02$.

Obviously, conditions leading to total reflection are to be avoided. For lossless materials this is equivalent to setting the transmission coefficient equal to zero; or according to (4) one has $B+C=0$. This may be reduced to the condition that

$\left(k^{2}-s^{\prime 2}\right)^{2} d^{4} \cos s^{\prime} d \sin s^{\prime} d$

$$
+4(k d)^{2} q^{\prime} d s^{\prime} d \cos q^{\prime} d \sin q^{\prime} d=0 .
$$

By general appearance this expression is similar to the dispersion relations for antisymmetric and symmetric Lamb waves $\left(\Delta_{a}=0\right.$ and $\left.\Delta_{s}=0\right)$ with $\Delta_{a}, \Delta_{s}$ given by (9) and (10). Equation (11) is not a dispersion relation, though, but a locus of points in the wave speed versus frequency plane for which zero transmission occurs.

This locus in terms of the relative wave speed $c / c_{s}$ versus the dimensionless frequency $k_{s} d$ is shown in Fig. 3. The vertical asymptotes, for which $c / c_{s} \rightarrow \infty$ and thus $k d \rightarrow 0$, are determined by $\cos s^{\prime} d \sin s^{\prime} d=0$ in (11). Therefore, the regions where large reflection coefficients are expected will be found near the thickness resonances $k_{s} d=n \pi / 2$ or $h / \lambda_{s}=$ $0.5 n$, with $n=1,2,3, \cdots$.

Notice that the curve in Fig. 3 illustrating (11) is a function of the plate material only. Its shape is determined by the value of $G / K$, or $\nu$. Of course, realization of wave reflection implies

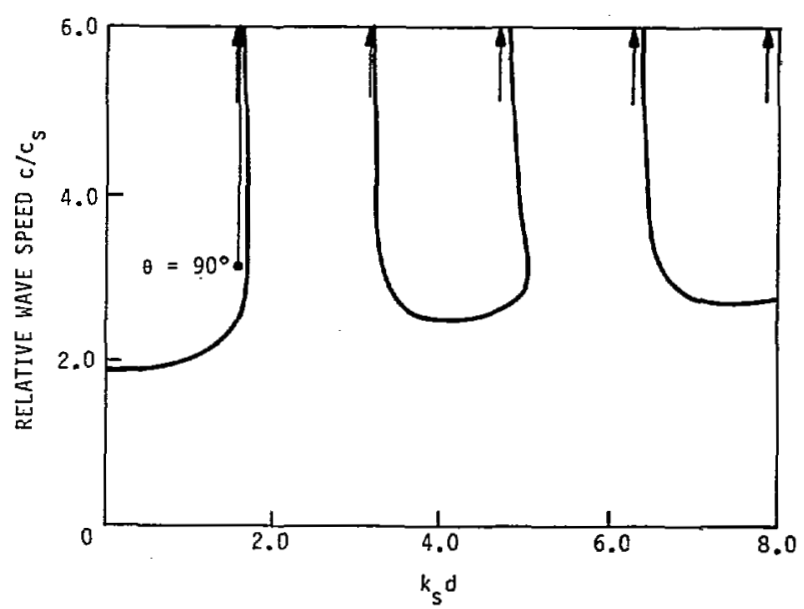

Fig. 3. Relative wave speed $c / c_{s}$ as a function of dimensionless frequency $k_{s} d$ for which zero transmission occurs. $G / K=0.13, \eta=0, \nu=0.438$. Arrows indicate asymptotes. The vertical line is the locus of points with $h / \lambda_{s}=0.51\left(k_{s} d=1.6022\right)$ and $\theta$ varying from $90^{\circ}$ to $0^{\circ}$.

interaction with a medium. For fixed frequency and variable angle the relative wave speed $c / c_{s}$ as a function of incidence angle $\theta$ is determined by the coincidence relation $c / c_{s}=c_{0} /\left(c_{s}\right.$ $\sin \theta$ ), and is represented by a vertical line in Fig. 3 starting at $\theta=90^{\circ}$, for which the coordinate $c / c_{s}$ is equal to $c_{0} / c_{s}$. The abscissa is $k_{s} d$, which is equal to $\pi h / \lambda_{s}$. 


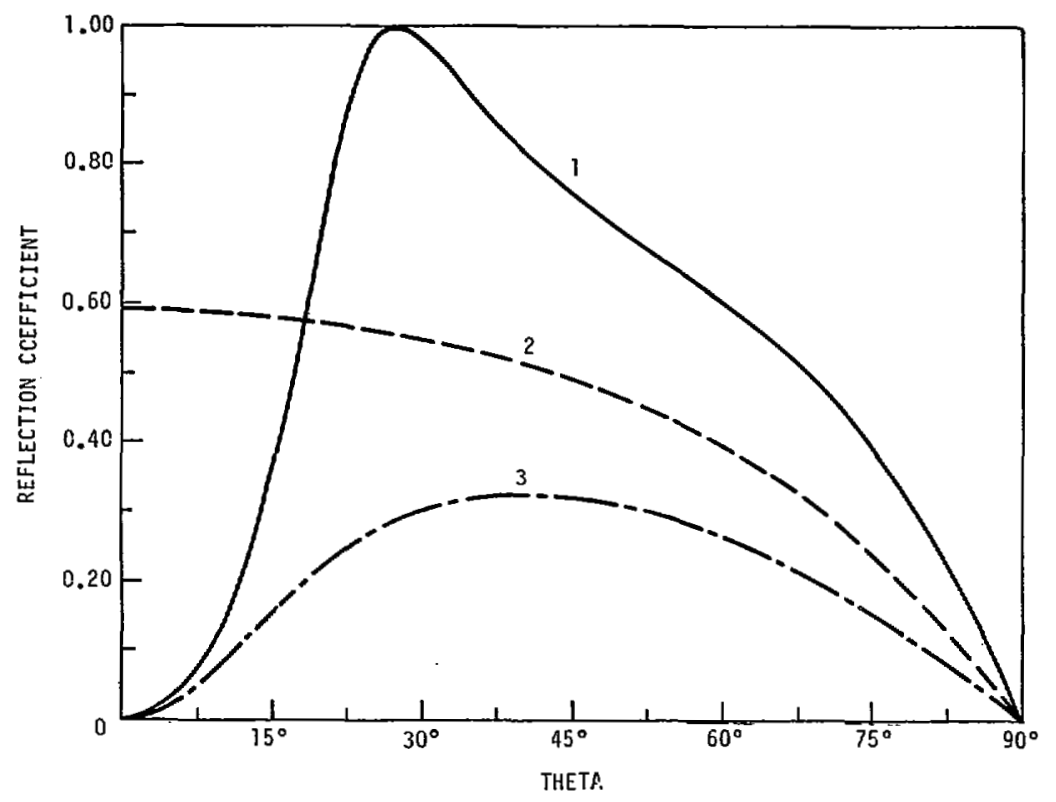

Fig. 4. Reflection coefficient as a function of incidence angle $\theta$ near first thickness-shear resonance. Ideal $\rho c, G / K=0.13, \eta=0, \nu=0.438$. $1-$ $h / \lambda_{s}=0.51 ; 2-h / \lambda_{s}=0.50 ; 3-h / \lambda_{s}=0.49$.

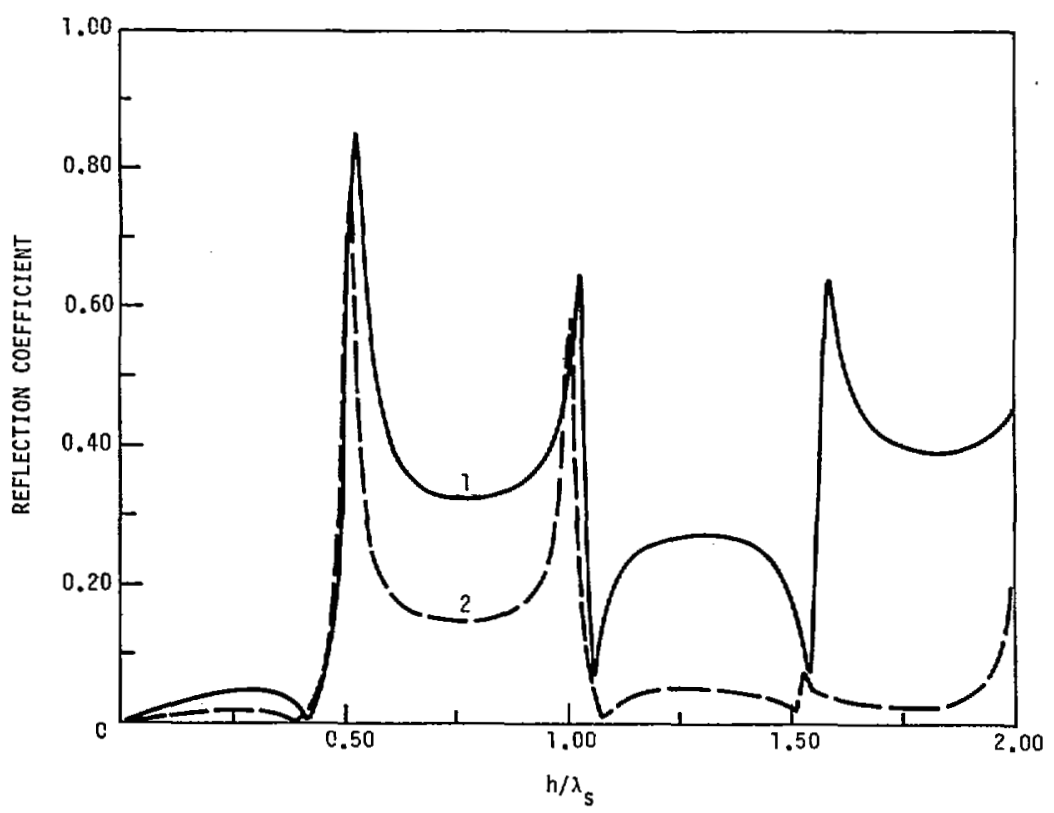

Fig. 5. Reflection coefficient as a function of thickness relative to shear wavelength $h / \lambda_{s}$. Ideal $\rho c, G / K=0.13, \eta=0.01, \nu=0.438+0.0006 i$. $1-\theta=60^{\circ} ; 2-\theta=30^{\circ}$.

Examples of the reflection coefficient as a function of incidence angle near a thickness-shear resonance are shown in Fig. 4, for ideal $\rho c$ material. The vertical line in Fig. 3 corresponds to a relative thickness $h / \lambda_{s}=0.51$, for which $k_{s} d=1.6022$ and $c_{0} / c_{s}=3.004$. The total reflection in Fig. 4 for $h / \lambda_{s}=0.51$ at $27.5^{\circ}$ corresponds to an intersection of the vertical line in Fig. 3 with the curve at $c / c_{s}=6.5$, just above the frame. For $\rho c$ material, one has $c_{0} \approx c_{d}$, and thus the starting points of the vertical lines for varying frequency lie on a horizontal line with $c_{0} / c_{s} \approx c_{d} / c_{s}=[2(1-\nu) /(1-$ $2 \nu)]^{1 / 2}$, which for $\nu=0.438$ is close to 3 . Thus, in the case of $\rho c$ material, the only realizable occurrence of total reflection is for values of $k_{s} d$ close to the asymptotes, at $n \pi / 2$ $(n=1,2, \cdots)$. When $h / \lambda_{s}$ approaches 0.5 , the total reflection peak becomes sharper and moves to zero incidence angle while still $R=0$ for $\theta=0$. For $h / \lambda_{s}<0.5$ there is no intersection of the corresponding vertical line with the curve in Fig. 3 and thus there is no angle at which total reflection occurs.

Another view on the thickness-resonances' effect on the reflection coefficient is given in Fig. 5, where the reflection is shown for fixed angles of $\theta=60^{\circ}$ and $30^{\circ}$ with varying relative thickness $h / \lambda_{s}$. The material is ideal $\rho c$, with $G / K=$ 0.13 and a loss tangent $\eta$ in $G$ of 0.01 . 


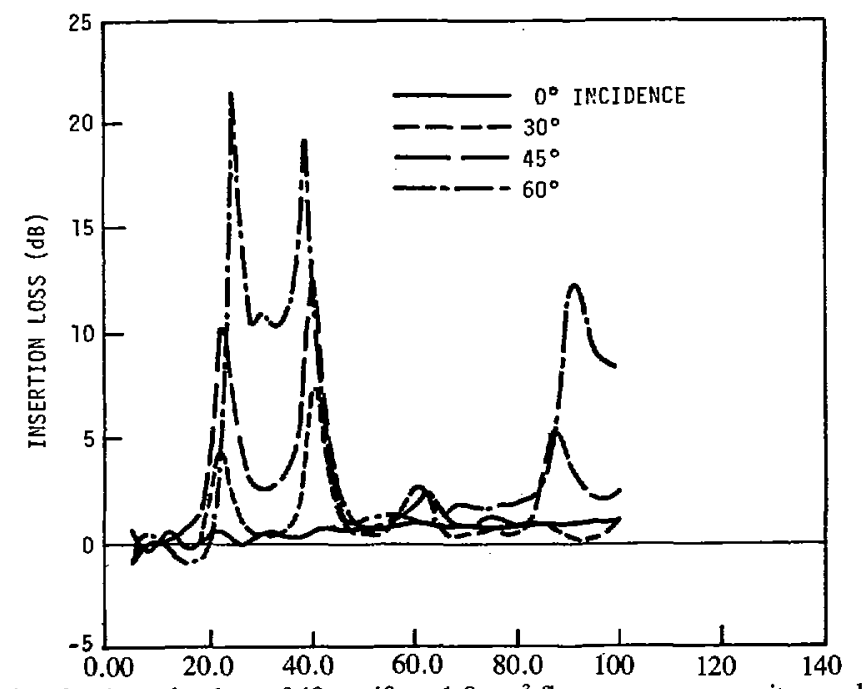

Fig. 6. Insertion-loss of $40 \times 40 \times 1.9-\mathrm{cm}^{3}$ fluoroepoxy composite panel at $22^{\circ} \mathrm{C}$ temperature and $345-\mathrm{kPa}$ pressure, as a function of frequency at various angles of incidence. Acoustic medium is freshwater, $\rho_{0} / \rho_{s}=$ $0.966 ; c_{0} / c_{d}=0.999$.

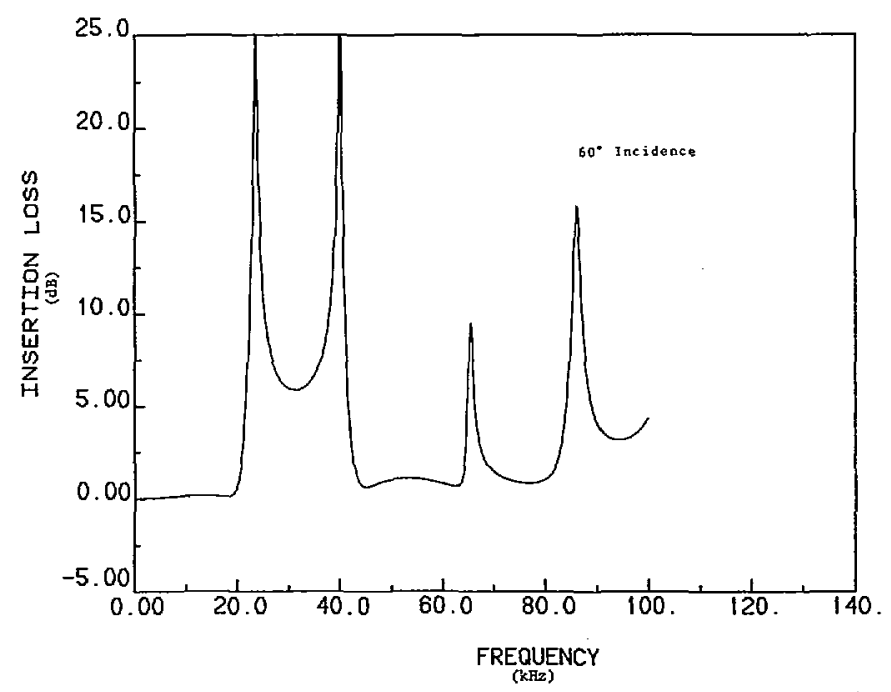

Fig. 7. Computation of insertion loss of a 1.9-cm-thick infinite plate as a function of frequency at $60^{\circ}$ angle of incidence. $G / K=0.35, \eta=0.01$; $\rho_{0} / \rho_{s}=0.966 ; c_{0} / c_{d}=0.999$.

\section{Comparison of InSERTION-Loss MEASUREMENT AND ANALYSIS}

Thompson and Griffith ${ }^{1}$ have reported on the measurement of insertion loss of a panel of structurally rigid $\rho c$ material as a function of frequency at several angles of incidence. The panel was $40 \times 40 \mathrm{~cm}^{2}$ square and $1.9 \mathrm{~cm}$ thick. Insertion loss is defined as 20 times the logarithm of the inverse absolute transmission coefficient. The results are shown in Fig. 6.

At $0^{\circ}$ incidence angle there should be no effect of shear waves; the wavy structure is tentatively ascribed to diffraction effects. At oblique angles of incidence one sees a welldeveloped system of peaks which one would be inclined to identify with the thickness-shear resonances described in the foregoing analysis.

${ }^{1}$ C. M. Thompson and J. R. Griffith reported on these results at the 111th Meeting of the Acoustical Society of America and intend to publish them. For the abstract, see J. Acoust. Soc. Amer. Suppl. 1, vol. 79, p. S62, Spring 1986.
To quantify the correspondence between theory and experiment, the shear modulus of the given material was measured by a method described by Adkins [8]. Earlier measurements suffered from a problem with the attachment of the sample to the cover plate, leading to the low value of 0.13 for $G / K$ used in Figs. 2-5. After correction of the problem, a value of 550 MPa was found for $G$. The bulk modulus was measured by means of an acoustic coupler [9]. Its value at $1000 \mathrm{~Hz}$ was found to be $1.55 \mathrm{GPa}$, giving a ratio $G / K$ of 0.35 .

Thompson and Griffith reported a value of $1033 \mathrm{~kg} / \mathrm{m}^{3}$ for the density of the material and a sound speed of $1490 \mathrm{~m} / \mathrm{s}$ at $1.5 \mathrm{MHz}$. From the experimental values for $G$ and $K$, and the reported density, one may compute a value for $c_{d}$ in the kilohertz region of $1487 \mathrm{~m} / \mathrm{s}$, indicating little dispersion in the dilatational wave speed.

The computed value for the insertion loss as a function of frequency for $G / K=0.35$, and $\eta=0.01$ is shown in Fig. 7, for an incidence angle of $60^{\circ}$. There is reasonable agreement with the experimental curve at $60^{\circ}$ incidence of Fig. 6 . Discrepancies in height and location of the peaks might be due to the finite size of the panel, and the finite angular resolution of the projector and hydrophone in the panel test.

\section{Conclusions and Recommendations}

This study shows the importance of thickness resonances in evaluating the effect of shear modulus on the behavior of structurally rigid $\rho c$ materials.

It is important, for frequencies higher than those for which the plate can be considered thin relative to the shear wavelength, to measure and, where necessary, to adjust the value of the shear modulus relative to the bulk modulus to avoid frequency regions where the thickness is close to an integer times half the shear wavelength.

For thick plates, the reflection coefficient becomes large for angles near about $60^{\circ}$ even when the frequency is not close to thickness-shear resonance.

\section{ACKNOWLEDGMENT}

The authors express their gratitude to Dr. C. M. Thompson and Dr. J. R. Griffith for making the results of the panel tests on structurally rigid $\rho c$ material available to this study.

\section{REFERENCES}

[1] R. E. Montgomery, F. J. Weber, D. F. White, and C. M. Thompson, "On the development of acoustically transparent structural plastics," $J$. Acoust. Soc. Amer., vol. 71, pp. 735-741, Mar. 1982; "Erratum," vol. 72, p. 635, Aug. 82.

[2] C. M. Thompson and J. R. Griffith, "Rigid, acoustically transparent plastic based on fluoroepoxy," presented at the 111th Meeting Acoust. Soc. Amer., Cleveland, OH, 1986; abstract published in J. Acoust. Soc. Amer. Suppl. 1, vol. 79, p. S62, Spring 1986.

[3] P. S. Dubbelday, "Influence of shear modulus on the behavior of acoustically transparent materials," Naval Res. Lab., Orlando, FL, Memorandurn Rep. 5745, Apr. 30, 1986.

[4] H. Reissner, "Der senkrechte und schräge Durchtritt einer in einem flüssigen Medium erzeugten ebenen Dilatations--(Longitudinal)--Welle durch eine in diesem Medium befindliche planparallelle feste Platte," Helv. Phys. Acta, vol. 11, pp. 140-155, 1938; "Erratum," vol. 11, p. $268,1938$.

[5] L. M. Brekhovskikh, Waves in Layered Media. Moscow, USSR: Acoustics Inst., Academy of Sci. USSR, 1973. Translation: R. T. 
Beyer, New York: Academic, 1980, pp. 70-81.

[6] P. S. Dubbelday, "Application of a new complex root-finding technique to the dispersion relations for elastic waves in a fluid-loaded plate," SIAM J. Appl. Math., vol. 43, pp. 1127-1139, Oct. 1983.

[7] I. A. Viktorov, Rayleigh and Lamb Waves. New York: Plenum, 1967.

[8] R. L. Adkins, "Design considerations and analysis of a complexmodulus apparatus," Exp. Mech., vol. 6, pp. 362-367, July 1966.

[9] J. Burns and P. S. Dubbelday, "Dynamic bulk coefficient measurements on several elastomers," in Proc. ONR/NUSC Symp. Dynamic Mechanical Properties of Elastomers (NUSC, New London, CT, July 1984), 1986, pp. 162-173.

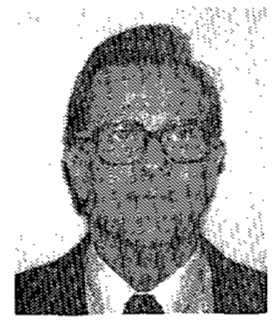

Pieter S. Dubbelday received the B.S. and M.S. degrees in physics and mathematics, and the Ph.D. degree in nuclear physics in 1959 from the Vrije Universiteit in Amsterdam, The Netherlands. In 1956, he did research at the Massachusetts Institute of Technology, Cambridge, under a fellowship and Fulbright grant.

He was a Research Associate and Instructor at the University of Wisconsin from 1959 to 1961 . From 1961 to 1966, he was a Senior Engineer with RCA, International Service Company, Patrick Air Force Base, FL, and from 1966 to 1981 he was Professor of Physics and
Oceanography at the Florida Institute of Technology in Melbourne, FL. Since 1981, he has been a Research Physicist with the Naval Research Laboratory, Underwater Sound Reference Detachment in Orlando, FL. His research interests are in elastic properties of elastomers, fluid-structure interaction, and the determination of particle velocity in hydroacoustic fields.

Dr. Dubbelday received the Teacher of the Year Award in 1980 and the Alan Berman Research Publication Award in 1984 and 1987. He is a member of the American Physical Society, a Fellow of the Acoustical Society of America, and serves on the ASA's Committee on Standards.

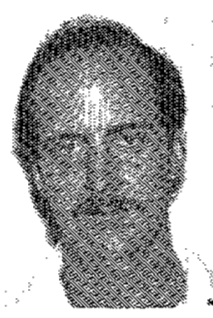

Kurt M. Rittenmyer was born in Cincinnati, $\mathrm{OH}$, on June 30, 1957. He received the B.S. degree in engineering from Harvey Mudd College, Claremont, CA, in 1979 and the Ph.D. degree in solidstate science from The Pennsylvania State University, University Park.

He joined the Underwater Sound Reference Detachment of the Naval Research Laboratory in Orlando, FL, in November 1983 and currently is a Research Physicist involved in the development of materials used for various underwater acoustic applications

Dr. Rittenmyer is an Affiliate of the IEEE and a member of the American Physical Society and the American Ceramic Society. 